\title{
SexTant: Visualizing Time-Evolving Linked Geospatial Data *
}

\author{
Konstantina Bereta ${ }^{1}$, Charalampos Nikolaou ${ }^{1}$, Manos Karpathiotakis ${ }^{2}$, \\ Kostis Kyzirakos $^{1}$, and Manolis Koubarakis ${ }^{1}$ \\ 1 National and Kapodistrian University of Athens, Greece \\ 2 École Polytechnique Fédérale de Lausanne, Switzerland
}

\begin{abstract}
We present SexTant, a Web-based system for the visualization and exploration of time-evolving linked geospatial data and the creation, sharing, and collaborative editing of "temporally-enriched" thematic maps which are produced by combining different sources of such data.
\end{abstract}

\section{Introduction and Motivation}

Linked geospatial data has recently received attention as researchers and practitioners have started tapping the wealth of geospatial information available in the archives of various national cartographic agencies and making it available on the Web as linked data [2]. As a result, in the last few years, the Web of data is being rapidly populated with geospatial information. As the real-world entities represented in linked geospatial datasets evolve over time, the datasets themselves get updated and both the spatial and the temporal dimension of data become significant for users.

In the demo paper [4] we presented Sextant ${ }^{3}$, a tool that enables the visualization and exploration of the spatial dimension of linked geospatial data. Sextant enables map creation and sharing, as well as the visualization and exploration of data by evaluating GeoSPARQL queries on SPARQL endpoints. In this way rich thematic maps can be created by layering information coming from the evaluation of GeoSPARQL queries. Sextant is based on standards defined by the Open Geospatial Consortium (OGC), thus it is interoperable with other well-known GIS and Web tools such as Google Earth.

In this demo paper we turn our attention to the temporal dimension of linked geospatial data and present a new version of Sextant that we now rename SexTant (the capital "T" in the new name emphasizes the time dimension). SexTant extends the functionalities of the earlier tool by visualizing the temporal dimension of data having a spatial extent simultaneously on a map and a timeline. The new capabilities of SexTant build on the temporal features of the data model

\footnotetext{
* This work was supported by the EU FP7 project TELEIOS (257662), the Greek NSRF project SWeFS (180), and the EU project Optique (318338).

${ }^{3}$ See http://wikipedia.org/wiki/Sextant for the explanation of the name.
} 
stRDF, the query language stSPARQL, and their efficient implementation in the geospatial RDF store Strabon [1]. stRDF and stSPARQL go beyond the OGC standard GeoSPARQL by allowing the representation and querying of linked geospatial data that changes over time [1,3]. SexTant (and this demo paper) extends the research presented in [1] by demonstrating how graphs defined in stRDF/stSPARQL can be explored and visualized.

In related work, the French project GEOPEUPLE is also studying the modeling and visualization of spatiotemporal data. As an example, the demo available at http://www.rotefabrik.free.fr/geopeuple/en/onglets-33038.html visualizes the evolution of administrative regions in France over the time.

\section{New Functionalities of SexTant}

SexTant extends the architecture of our earlier system presented in [4] and shown in Fig. 1a as follows (new and modified components are highlighted with pink boxes). First, apart from the the map ontology used by the earlier system and shown in Fig. 1b, SexTant employs the temporal ontology dictated by stRDF and stSPARQL for the modeling of valid time [1]. This ontology enables the introduction of user-defined time and valid time of a triple in stRDF data. Times are modelled as instants or intervals and are represented using values of the datatypes xsd:dateTime and strf:period respectively. Second, one can now use all the temporal features of stSPARQL to query linked spatiotemporal data encoded in stRDF. In this way the full capabilities of endpoints using the spatiotemporal RDF store Strabon can be exploited. Third, the module that translates the results of stSPARQL queries from XML to KML format has been extended so that the temporal primitives of stRDF that we mentioned above are translated into the respective temporal primitives of the KML standard. An example of this transformation is provided in Fig. 1c. Last, SexTant builds on the Timemap Javascript library (https://code.google.com/p/timemap/) for visualizing "temporally-enriched" KML files. This enables the visualization of geospatial features with associated temporal information on a map and a timeline simultaneously. Timemap has been transparently integrated in the implementation of the earlier system which is based on the Google Web Toolkit framework.

\section{Demonstration Overview}

The demonstration of the spatio-temporal features of SexTant will be based on a real scenario in which an Earth Observation (EO) scientist studies the changes in the land cover of an area and assesses the damage caused by fires. This scenario is very common in the EO domain, where data is constantly produced by satellite sensors and is associated with metadata containing, among others, temporal attributes, such as the time that an image was acquired. Satellite acquisitions are utilized in related applications such as the CORINE Land Cover 


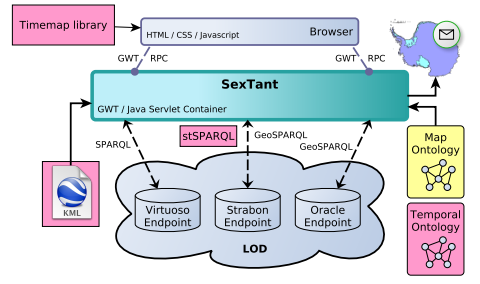

(a)

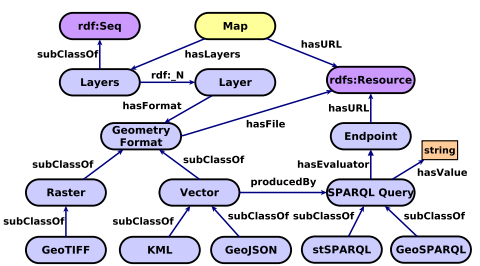

(b)

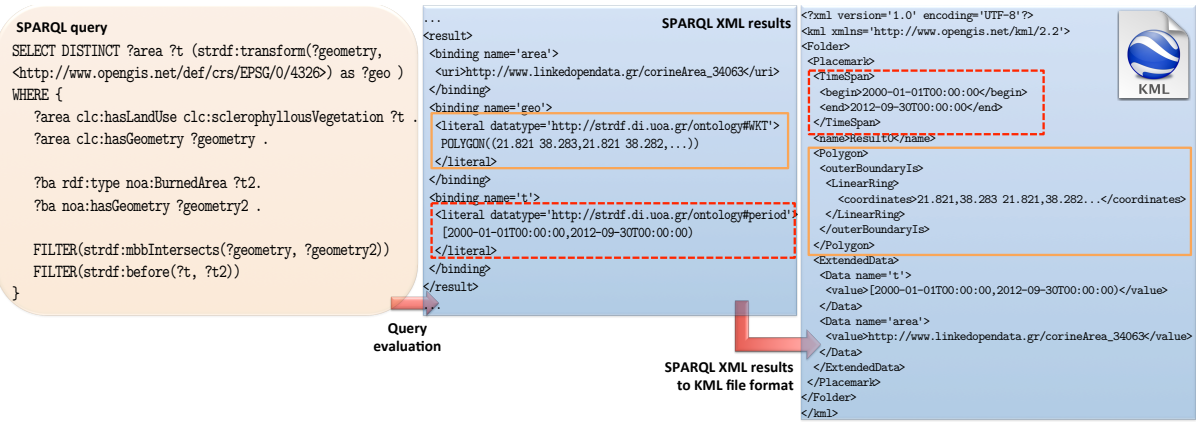

(c)

Fig. 1: (a) SexTant overview and (b) Map ontology (c) Translation of SPARQL XML results to KML

programme operated by the European Environment Agency that makes available as a cartographic product the land cover of European areas over time.

To achieve the goal of our scenario, we will combine information derived from the following datasets that were produced within the project TELEIOS (http: //www. earthobservatory.eu/): the CORINE Land Cover dataset of year 2000, a Fire Hotspots dataset that provides information about fire hotspots in Greece, and a Burned Areas dataset that provides detailed information about areas of Greece that have been affected by fires during a recent fire season. The EO scientist will use SexTant to visualize the results of stSPARQL queries that use several thematic, spatial and temporal criteria so that she will be able to derive implicit links among the involved datasets due to their spatial and temporal correlation.

In our scenario, first we will visualize on a map the areas that have been classified as "sclerophyllous vegetation" according to the CORINE Land Cover dataset of year 2000. The valid time of the triples that encode information about these areas will be projected on the timeline. Next, a new layer that visualizes the hotspots that have been identified during the fire season will be displayed on the map while the timeline will display the time when the hotspots were detected. Then, a new layer that depicts the areas that were burned during the forest fires of 2012 will be overlayed on the map and the timeline. The resulting map will display to the EO scientist the schlerophyllous forests that got burnt by 


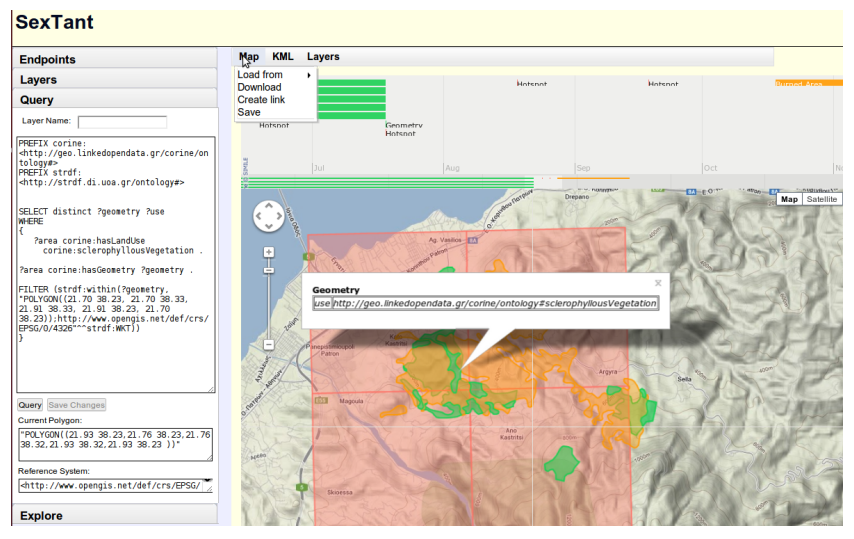

Fig. 2: A screenshot from SexTant depicting the evolution of the land cover

the forest fires of 2012 along with a preview of the evolution of the forest fires as they were detected by satellites so that she can assess the severity of the damage caused by fires. A similar procedure will be used in order to discover implicit links among the datasets enriched with provenance information, e.g., discover the cause of changes in the land cover of areas as represented in CORINE through the visualization and overlay of the other datasets. Such implicit links can later on be asserted to enrich all datasets.

Layers that contain solely geospatial information will be retrieved by evaluating a GeoSPARQL query on a Strabon, Oracle, Parliament, or Virtuoso endpoint, while layers that contain spatial and temporal information will be retrieved by evaluating an stSPARQL query on Strabon. The reason for this choice is that stSPARQL is the only language that provides the spatial and temporal primitives that are needed for this scenario, while Strabon is currently the only "temporally-enabled" geospatial RDF store as we have discussed in [1]. In this respect our demo will also serve to showcase the new functionalities of the system Strabon as presented in [1]. A video demonstration of SexTant that follows the scenario described in this section is available at http://strabon.di.uoa.gr/sexTant/sexTant-demo-full-version.ogv.

\section{References}

1. Bereta, K., Smeros, P., Koubarakis, M.: Representation and querying of valid time of triples in linked geospatial data. In: ESWC. LNCS, vol. 7882, pp. 259-274 (2013)

2. Koubarakis, M., Karpathiotakis, M., Kyzirakos, K., Nikolaou, C., Sioutis, M.: Data Models and Query Languages for Linked Geospatial Data. LNCS, vol. 7487, pp. 290-328. Springer (2012)

3. Kyzirakos, K., Karpathiotakis, M., Koubarakis, M.: Strabon: A Semantic Geospatial DBMS. In: ISWC. LNCS, vol. 7649, pp. 295-311. Springer (2012)

4. Nikolaou, C., Dogani, K., Kyzirakos, K., Koubarakis, M.: Sextant: Browsing and Mapping the Ocean of Linked Geospatial Data. ESWC 2013. Demo paper 